\section{Fitting a Student Microscope with a Consumer Digital Camera}

Theodore M. Clarke, Metallurgical Failure Analysis Consultant tclarke@ligtel.com

My home microscopy of lake water organisms with my two student microscopes lead to my active participation in Micscape Internet magazine avallable at the web site http:// www.microscopy-ukimag. An article in Micscape by Dave Walker, http://www. microscopy-uk.org.uk/mag/artjan02/toptips8.html, played a critical role in my decision to purchase a Nikon Coolpix 995 for my home microscopy. Dave's article referenced a far more thorough article on fitting a consumer digital camera to older style microscopes: The New Microscopy by Peter Evennett, Proceedings RMS, Vol. 35 (4), Dec 2000. Anyone seriously interested in this subject should read Peter's article available from the RMS web site at http://www.rms.org.uk. According to Peter Evennett's article, Nikon supplies mounts for use of the Coolpix on their microscopes with fully corrected primary images. My first student microscope is an upgraded Monolux fitted with achromatic objectives not requiring a compensating eyepiece. My second student microscope is a LOMO Biolam, which uses a compensating eyepiece system. This article will show the mounting system I made to use the Coolpix 995 on each microscope with primary emphasis on the Biolam and how the full field of a $10 \times 18 \mathrm{~mm}$ FN compen- sating type eyepiece can be recorded without vignetting. The Coolpix, with its internal focusing zoom lens of moderate maximum aperture, may be unique in its ability record a photomicrograph without vignetting the field seen through an appropriate high eyepoint eyepiece. My camera mount supports the camera through its sturdy tripod mount and contains a cable release to avoid vibration. My prior work experience with digital imaging was. with the scientific grade monochrome MegaPlus 1.6i/AB camera, which does not have a color mosaic filter on the sensor. My experiments had indicated that the 1.6 megapixels of the monochrome camera were adequate to replace film imaging with an 18 $\mathrm{mm}$ diameter primary image and that a 3.2 megapixel color mosaic sensor would be needed to provide the same spatial resolution. Resolution testing of the Coolpix 995 confirmed this expectation and that resampling with Adobe Photoshop of its $2048 \times$ 1536 pixel image to a $1434 \times 1075$ pixel image could be done without significant loss in spatial resolution.

I made the mount for the Coolpix from aluminum, see Figure 1. A lug on the base plate supports an arm for the cable release. The lug is tapped with a $1 / 4^{\prime \prime}-20$ thread so it can be attached to a camera stand to eliminate need for the eyepiece bushing which attaches to the base with \#2-56 screws. Figure 2 shows the Coolpix mounted to its base plate on my photomacrography stand beside the $10 \mathrm{X}$ high eyepoint eyepiece in my modified Monolux. (The non-compensating high eyepoint eyepiece

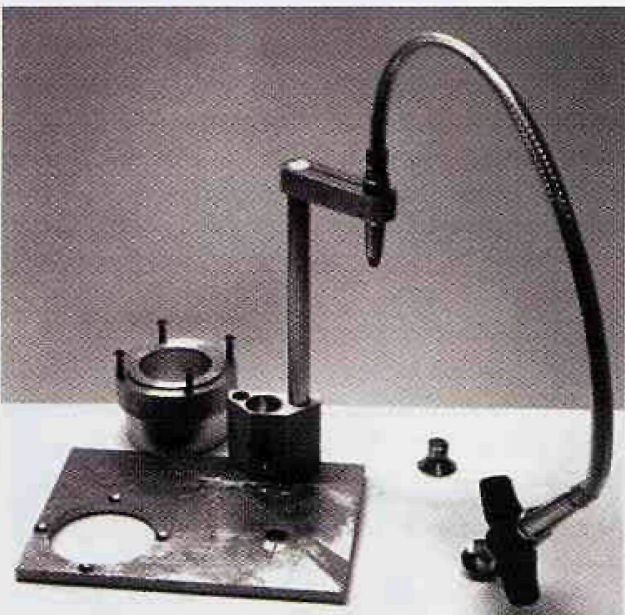

Figure 1: Microscope mounting bracket and eyepiece bushing for a Nikon Coolpix camera.

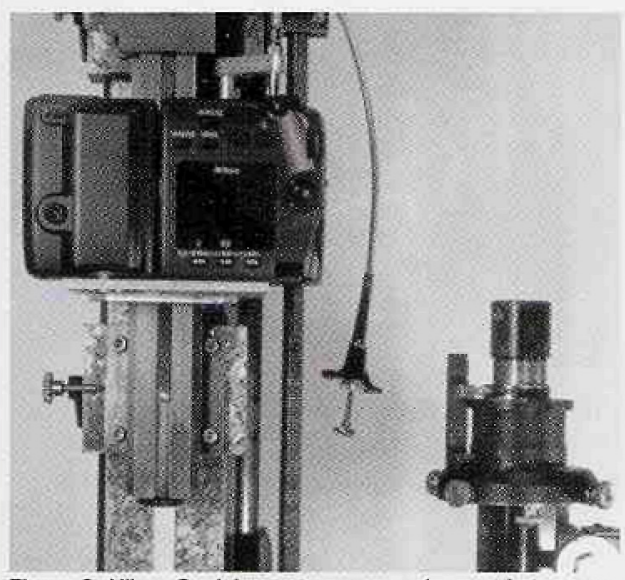

Figure 2: Nikon Coolpix camera mounted on a photomacrography stand with the bottom of the mounting base flush with the top of the high eyepoint eyepiece in a monocular Monolux microscope.

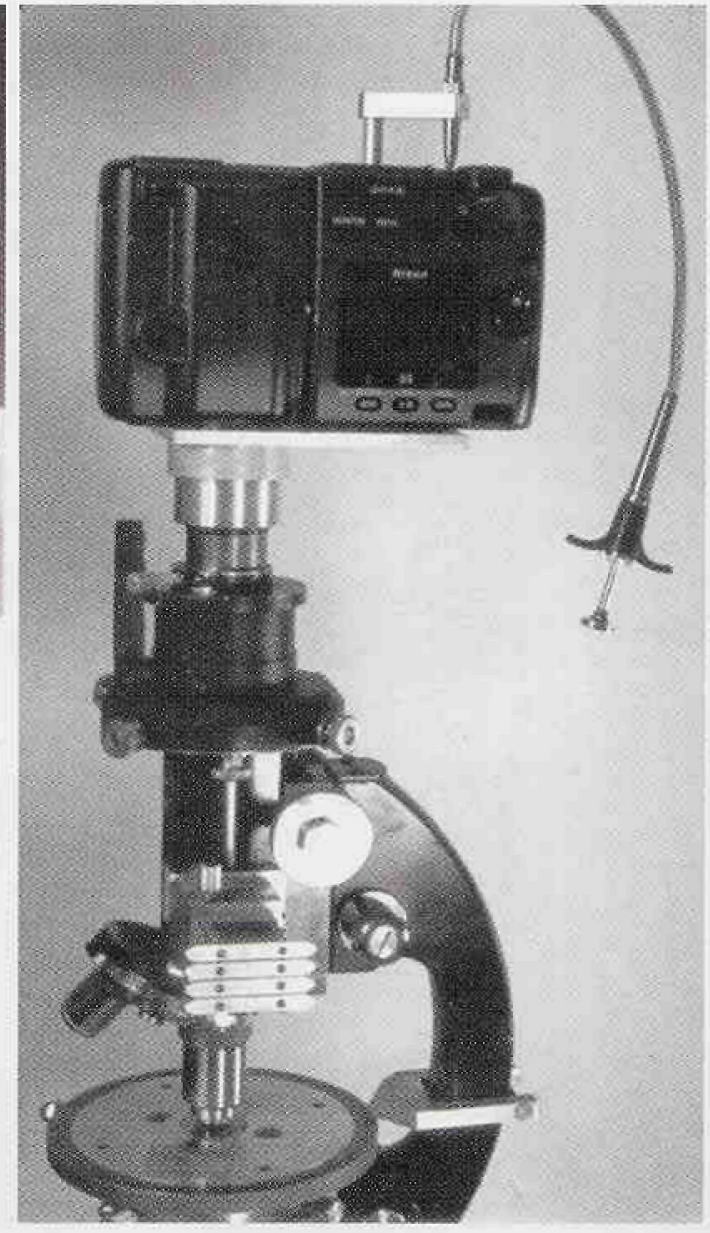

Figure 3: Polarized light capable Monolux microscope with a Nikon Coopix camera mounted on the eyepiece.

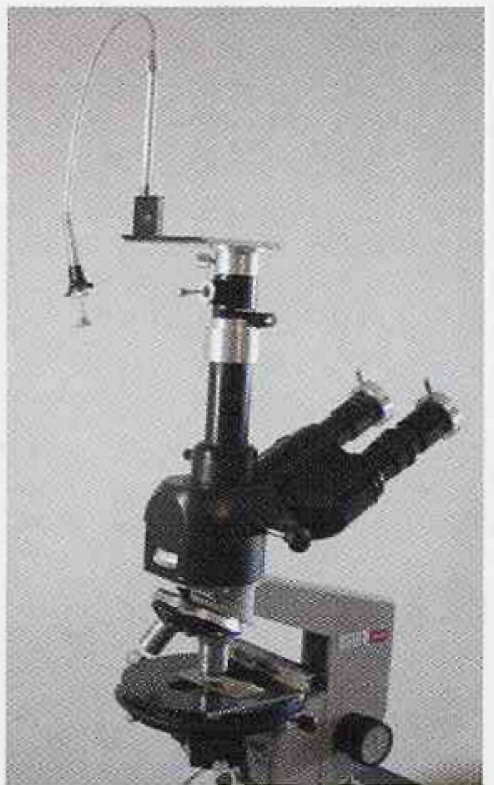

Figure 4: Mounting bracket for a Nikon Coolpix camera attached to a LOMO Edupointer evepiece in the trinocular phototube on a LOMO Biolam microscope with eyecaps on the binocular eyepieces to correct for the user's astigmatism and nearsightedness.

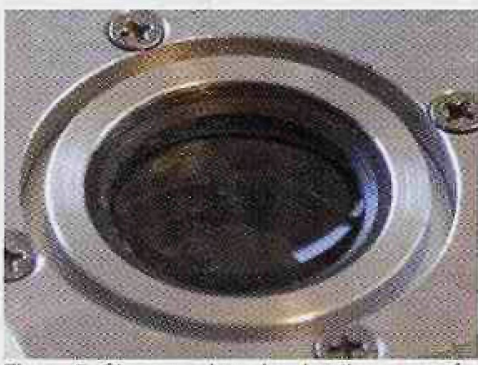

Figure 5: Close-up view showing the recess for the Coolpix camera lens flange to minimize the eyepiece to lens gap. 
was obtained from Mark Simmons. Mark custom makes adapters for mounting the Coolpix and other digital cameras to microscopes and can be reached through his e-mail address: marksimmons@rocketmail.com.) The image field is selected and focused, while wearing prescription eyeglasses, just prior to sliding the microscope under the camera for digital imaging. Working the camera controls does not transfer forces to the microscope, which is an advantage resulting from not attaching the camera directly to the microscope. I have found that the camera autofocus does not work properly for this application and that the camera focus must be set at infinity. Younger microscopists may have trouble relaxing their vision so that the eyepiece image is viewed at infinity. It is possible to feed the video output from the camera to a monitor to focus the microscope with the camera zoomed in for resolving finest detail prior to zooming out for recording. This is the method recommended by Vishnu Reddy in his Micscape article found at http://www. microscopy-uk.org.uk/mag/artaug01/ vrcoolpix.html. His mounting system also attaches to the camera tripod mount because of concern about loading the threads of the camera lens flange with the more common method of using an adapter bushing over the eyepiece threaded into the lens flange. Bumping the camera could overload this critical region. Figure 3 shows the Coolpix mounted to the eyepiece in the Monolux. I do not recommend this approach on a monocular system because working the camera controls could affect the microscope focus. This direct mounting approach would work with the camera mounted to one of the eyepieces of a binocular head, which would be the most common use. The field curvature with the simple achromatic objectives used on the Monolux limits the maximum primary image diameter to about $12 \mathrm{~mm}$ for decent quality images, which I would recommend be resampled from to $1433 \times 1075$ pixels prior to cropping.

I prefer the LOMO Biolam, with a trinocular head, for microscopy of live organisms in lake water samples. I normally use high intensity darkfield illumination from a fiber optic illumination system shown in my Micscape article found at http://www.microscopy-uk/ mag/artapr01/tclarke1.html. All my image recording of organisms has been on film, but now I am looking forward to the advantages of digital recording with the Coolpix with the mounting system on the photo tube of the LOMO trinocular head shown in Figure 4. Mounting the Coolpix on the Biolam HT-30 trinocular head and obtaining good images requires an understanding of its compensating type eyepiece system, which I learned by trial. I first used the short mount LOMO objectives in my Monolux monocular microscope and found they yielded very good quality images when used with a high eyepoint $12.5 \times$ Zeiss $\mathrm{kpl}$ eyepiece with an 18 $\mathrm{mm}$ field number. This same Zeiss eyepiece when used in the trinocular head shows blurring towards the edge of the field with the $40 \mathrm{X}$ objective. The reason for this is that the magnification changer lenses in the trinocular head correct for field curvature with the LOMO objectives. The LOMO $10 \times 18 \mathrm{~mm}$ field number eyepieces perform very well in the trinocular head because they compensate only for chromatic difference of magnification. The Zeiss kpl eyepiece corrects for both field curvature and chromatic difference of magnification. The LOMO $10 \mathrm{X}$ eyepieces are not high eyepoint, so I made eye caps with my corrective prescriptions for astigmatism and near sightedness, a method suggested in older Zeiss literature. The thumbscrews shown in the caps in Figure 4 are at the 12:00 position for correct alignment of the astigmatism axes. I also determined that the LOMO Edupointer $10 \mathrm{X}$ eyepiece has an $18 \mathrm{~mm}$ high eyepoint but adds $22 \mathrm{~mm}$ to the system tube length. The LOMO VA-E2 photo eyepiece tube adapter required an extension bushing for the Edupointer eyepiece to be parfocal with the eyepieces in the binocular viewing head. The Edupointer eyepiece had to be deeply recessed into the threaded flange of the Coolpix lens to avoid vignetting, see Figure 5. Figure 6 shows that the Coolpix is capable of imaging the full field of view of the Edupointer eyepiece with the $40 \mathrm{X}$ objective. The field diaphragm was set to so that the outer corners were seen at the edge of the field in the $10 \times 18-\mathrm{mm}$ field number eyepieces. The pointer tip still shows, even in the fully retracted position. Figure 7 shows that there is no vignetting when the Coolpix lens is zoomed in so the corners of the field diaphragm just show. The pointer could be very useful for demonstrating features in video output from the camera. This trinocular head is not suitable for polarized light applications because there is no provision for an analyzer and compensating wave plates. I made a housing for an analyzer and wave plates for use

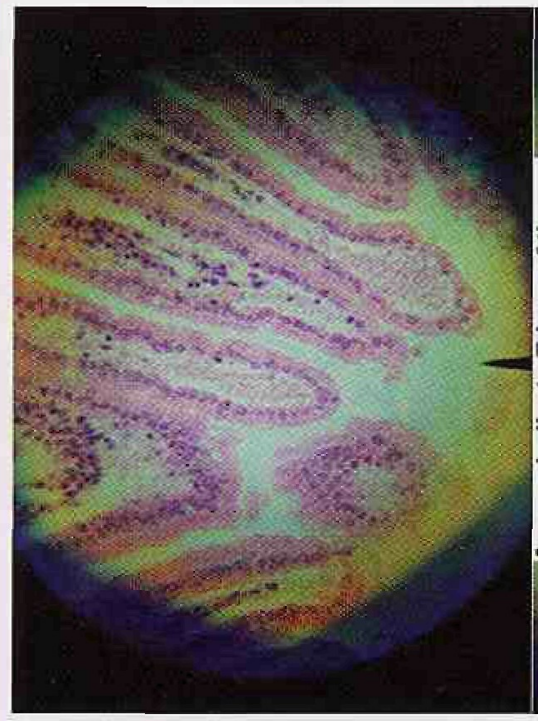

Figure 6: Coolpix camera lens zoomed out to show the full field of view of the microscope with the field diaphragm set to be just visible at the edge of the field of the $18 \mathrm{~mm}$ field number of the binocular viewing eyepieces

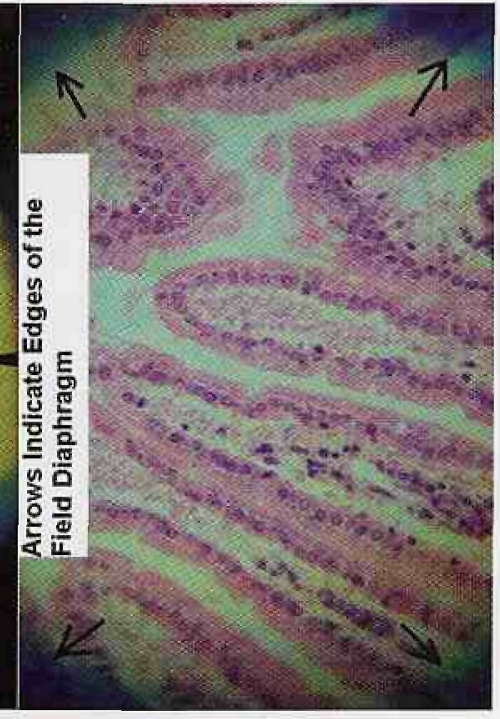

Figure 7: Same field as in Figure 6 after the Coolpix camera lens zoomed in until the edgas of the field diaphragm are just visible in the corners of the image with no evidence of vignetting from zooming

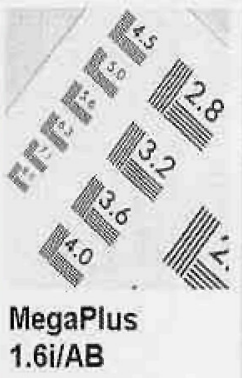

$60 \mathrm{~mm}$ Micro NIkkor Lens

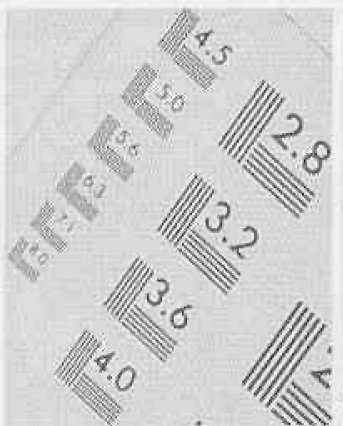

Nikon Coolpix 995 3.4 Megapixel File

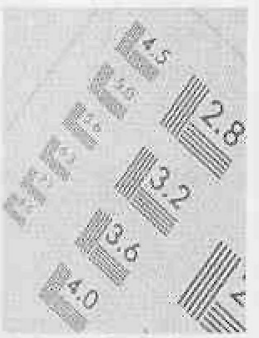

Nikon Coolpix $99570 \%$ Downsized to 1.6 Megapixels

\section{DIGITAL CAMERA RESOLUTION TESTS USING NATIONAL BUREAU OF STANDARDS-1963-A MICROCOPY RESOLUTION TEST CHART IMAGED IN AN 89 BY $133 \mathrm{~mm}$ FIELD SIZE}

Figure 8: Resolution test. (The author's figure was reduced $15 \%$ horizontally to fit the column wioth Editor.) 

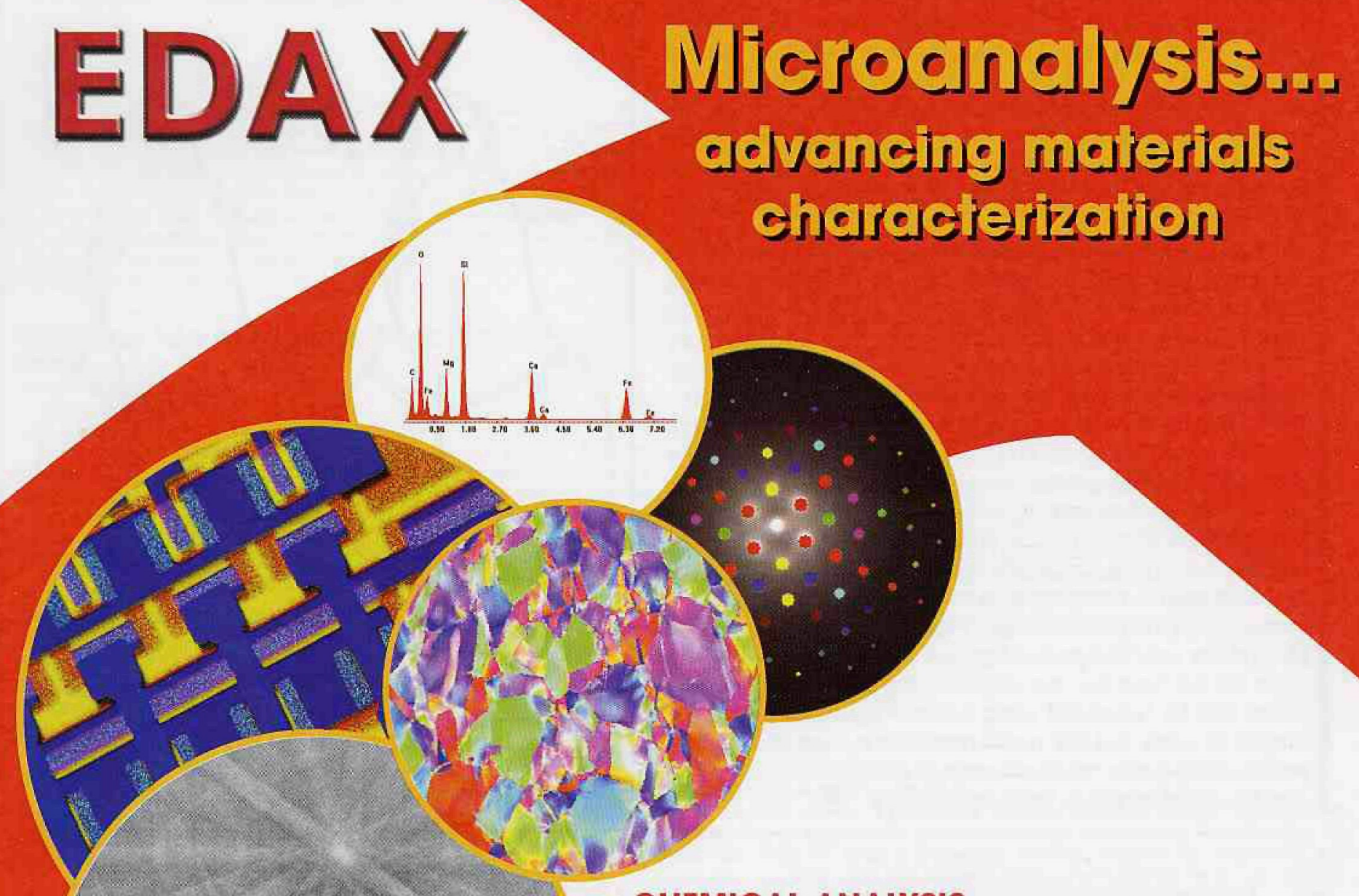

\section{CHEMICAL ANALYSIS}

- EDS in the SEM and TEM

GENESIS is the next generation of EDS analytical software on the Phoenix and Falcon platforms

- Microcalorimetry in the SEM

Polaris sets a new resolution standard for elemental analysis

\section{STRUCTURAL ANALYSIS}

- EBSD in the SEM

OlM provides crystal orientation mapping and texture analysis

Delphi combines EBSD and EDS data for phase identification

\section{INTEGRATED SOLUTIONS}

- Realize the next level of materials characterization with combined EBSD and EDS analysis 
Biolam, but the viewing system is monocular. Zooming in the Coolpix with a Zeiss $12.5 \times$ high eyepoint eyepiece vignettes the primary image field diameter to $14 \mathrm{~mm}$ in a setup equivalent to that shown for the Monolux in Figure 2. The $1.1 \mathrm{X}$ tube factor from the trinocular head is not present in the monocular application, so this is equivalent to a $15 \mathrm{~mm}$ primary image field diameter with the trinocular system. A $10 \times$ Zeiss $\mathrm{kpl}$ high eyepoint eyepiece should not have this limitation, but this is not a common lens.

I no longer have access to the Applied Image Inc. chrome on glass resolution test pattern used in my earlier research. Therefore I had to be content with testing the Coolpix spatial resolution with a test pattern based upon the NBS Microcopy Resolution Test Chart noted in my article "Resolution of Digital Photomicrographs from Scanned Film" in MICROSCOPY TODAY, Feb/March 2001. The resolution test pattern was cut from an office copier test sheet and has a finest line spacing of 8 line pairs per mm. This pattern was imaged in an 89 by $133 \mathrm{~mm}$ field size of a small format photographic print. A photomicrograph with an $89 \times 133$ image area printed at a magnification of 500 times the numerical aperture of the objective would be expected to yield a closest pattern spacing of 6 line pairs per $\mathrm{mm}$ measured on the printed image. Figure 8 shows that the Coolpix and the scientific grade Megaplus 1.6i/AB camera both resolve the 5.6 lines per $\mathrm{mm}$ pattern in this test and the Coolpix image can be resampled using Adobe PhotoShop to half the number of pixels and this spatial resolution is retained. The test pattern images may not reproduce well when published. I will email the digital version to those requesting it.

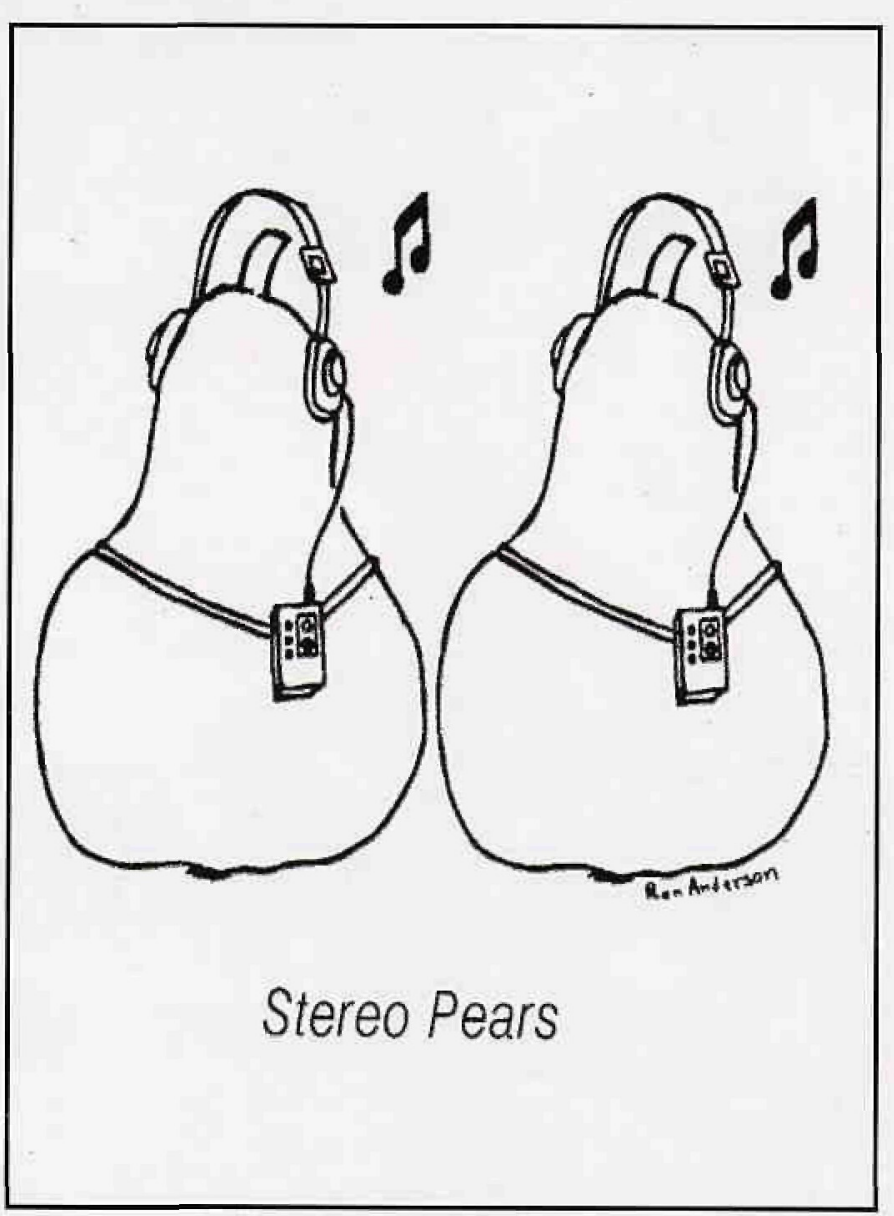

\section{Introducing EDS2000i, The Most Advanced Microanalysis System Today!}

- $100 \%$ Integrated inside the SEM operating system

- Apply the EDS tools on the live SEM Image

- Analyze samples, faster, with greater accuracy

- Save all EDS data along with the SEM image in one file

- Integrated Stage control for advanced particle analysis
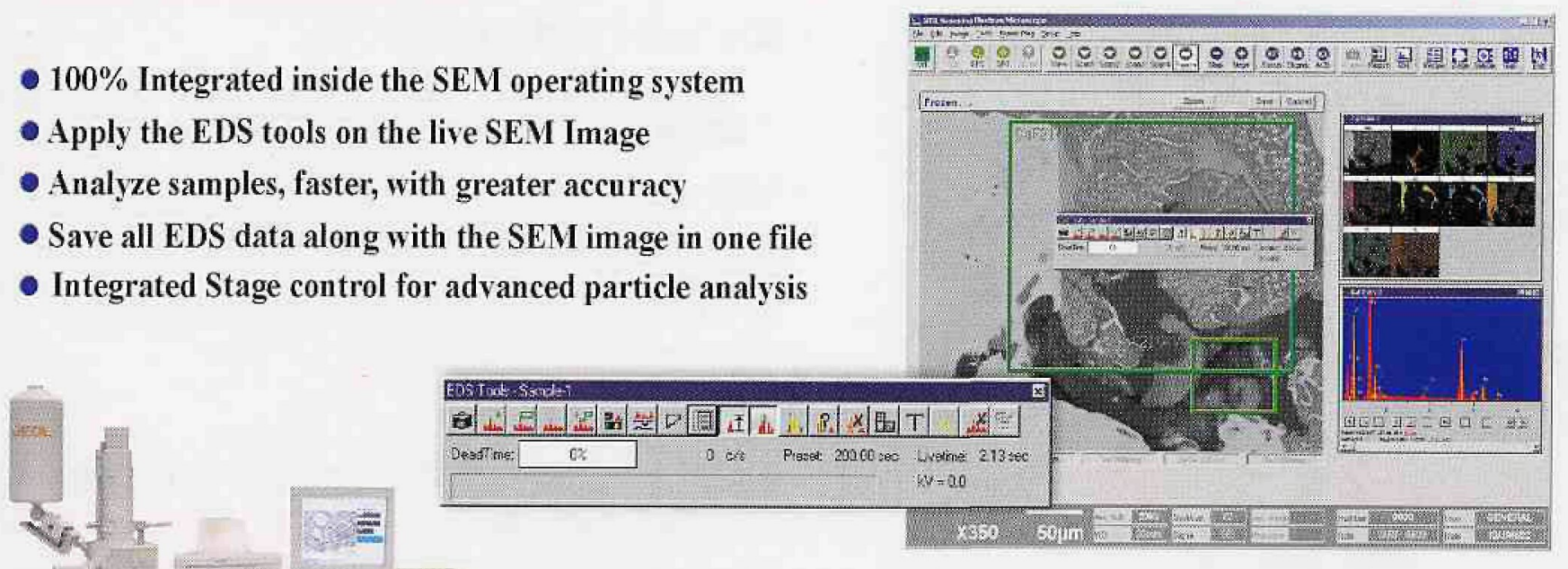

IXRF's Integrated EDS systems combine the SEM and IXRF EDS user interfaces into a single application. The EDS Toolbar is accessed directly from the SEM user interface allowing advanced microanalysis features to be performed directly on the live SEM image. Spectra, X-ray Maps, and Linescans can be directly acquired from the selected regions of the SEM image with a single click on the EDS Toolbar. The EDS and SEM user interfaces combine to provide one seamless microanalysis tool.

IXRF Systems, Inc., Houston, TX, USA, www. Ixrfsystems.com Ph:(281) 286-6485 Fax:(281)286-2660 


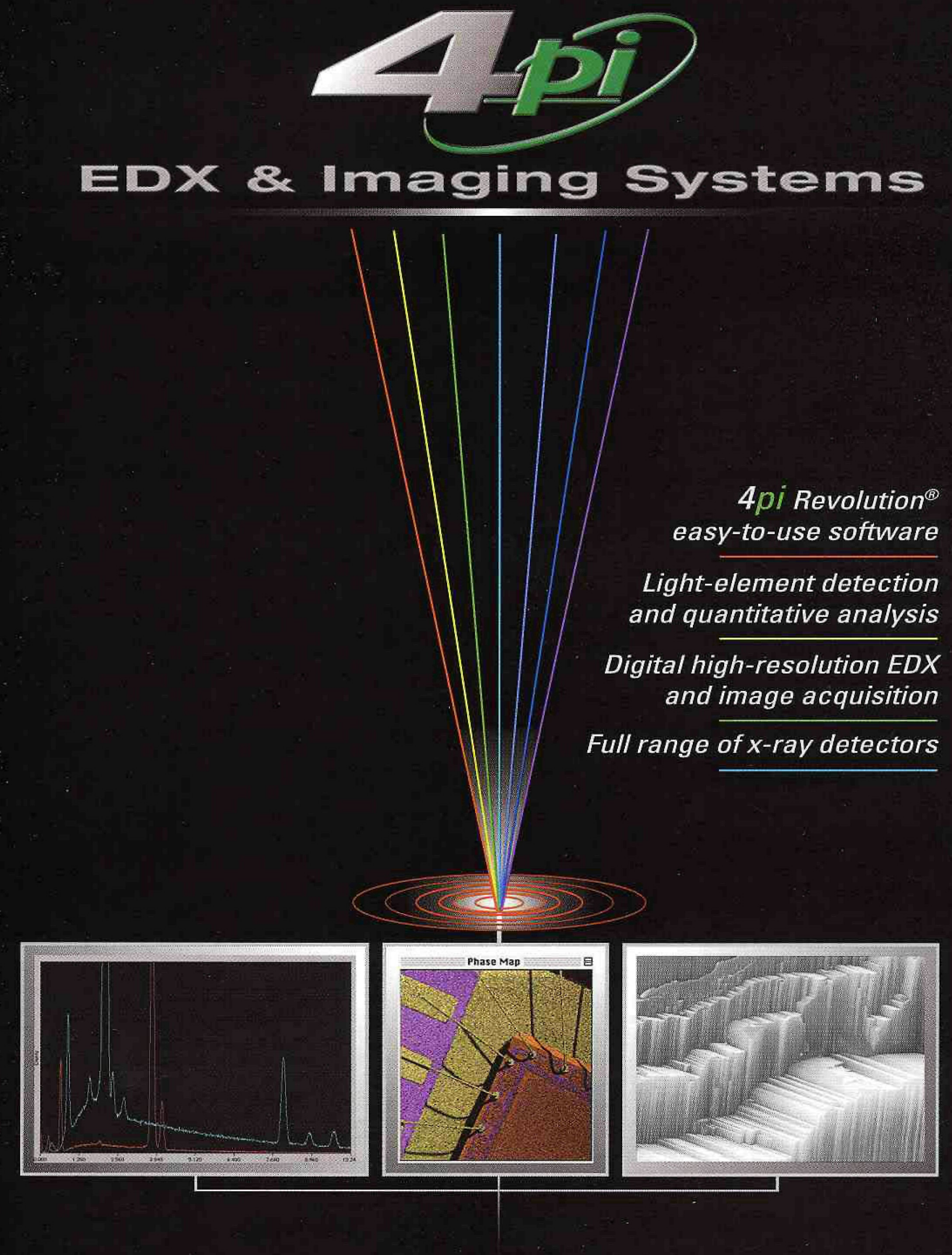

